\title{
Surface Region of Superfluid Helium as an Inhomogeneous Bose-Condensed Gas
}

\author{
A. Griffint and S. Stringari \\ Dipartimento di Fisica and INFM, Università di Trento, I-38050 Povo, Italy
}

(submitted to PRL, May 17, 1995)

\begin{abstract}
We present arguments that the low density surface region of self-bounded superfluid ${ }^{4} \mathrm{He}$ systems is an inhomogeneous dilute Bose gas, with almost all of the atoms occupying the same single-particle state at $T=0$. Numerical evidence for this complete Bose-Einstein condensation was first given by the many-body variational calculations of ${ }^{4} \mathrm{He}$ droplets by Lewart, Pandharipande and Pieper in 1988. We show that the low density surface region can be treated rigorously using a generalized Gross-Pitaevskii equation for the Bose order parameter.
\end{abstract}

PACS numbers: 05.30.Jp, 67.40.-w, 03.75Fi

In recent years, there has been a major effort to produce atomic-like gases in a Bose-condensed phase, using excitons, spin-polarized Hydrogen and laser-trapped alkali atoms (for reviews of this work, see Refs. [1,21). In such systems, the fraction of atoms in the lowest energy single-particle state could approach $100 \%$ at $T=0$. This unique phase of matter was first studied by Einstein and London and would exhibit many unusual properties [2], including superfluidity. In contrast, in bulk superfluid ${ }^{4} \mathrm{He}$, the condensate fraction is only about $10 \%$ at $T=0$ [1.,3]. Thus the ground state wavefunction does not have the simplicity of a gas with complete Bose-Einstein condensation (BEC).

In the present letter, we argue that such a dilute Bosecondensed gas is in fact already present in the surface region of superfluid ${ }^{4} \mathrm{He}$, where the density becomes very small. This striking phenomenon has apparently been overlooked in the extensive theoretical literature on surfaces, films and droplets of superfluid ${ }^{4} \mathrm{He}$ (for references, see Ref. 仼). Moreover, in this low density surface region, where one has almost $100 \%$ BEC into a single state, one can do microscopic calculations of the order parameter using the standard field-theoretic description of an inhomogeneous Bose-condensed fluid [5,6]. Our discussion is limited to ground state properties $(T=0)$ but we make a few remarks about finite temperature at the end.

Striking confirmation of the preceding physical argument is available in the results of Lewart, Pandharipande and Pieper (LPP) [7], who studied ${ }^{4} \mathrm{He}$ droplets (with up to 240 atoms) using a variational ground-state wavefunction approach [8]. These authors calculated the singleparticle "natural orbitals" $\psi_{i}(\mathbf{r})$ which diagonalize the single-particle density matrix, giving

$$
\rho_{1}\left(\mathbf{r}, \mathbf{r}^{\prime}\right)=\sum_{i} n_{i} \psi_{i}^{*}(\mathbf{r}) \psi_{i}\left(\mathbf{r}^{\prime}\right),
$$

where $n_{i}$ is the occupation probability of the state $\psi_{i}(\mathbf{r})$. The local density is given by $\rho(\mathbf{r})=\rho_{1}(\mathbf{r}, \mathbf{r})$. By direct numerical calculation, LPP found that the node-less $1 s$ state $\psi_{1 s}(\mathbf{r})$ had an occupation probability of $n_{1 s} \simeq 25$, while for all the other states, $n_{i}<0.5$. They identified (and justified) this $1 s$ state as the Bose-condensed state, with an associated local condensate density being given by

$$
\rho_{c}(\mathbf{r})=n_{1 s}\left|\psi_{1 s}(\mathbf{r})\right|^{2} .
$$

LPP evaluated the total local density $\rho(\mathbf{r})$ and the condensate local density $\rho_{c}(\mathbf{r})$ using (2). Their results for $N=70$ atoms are replotted in Fig. 1. However, LPP did not explicitly discuss the implications of the most striking feature shown in Fig. 1, namely that since in the surface region almost all the atoms at a given value of $r$ are in the $\psi_{1 s}(\mathbf{r})$ state, this surface region provides a physical realization of the long sought-for dilute Bose-condensed gas [1.2].

An important aspect of the calculations in Ref. [7] is that near the center of the drop, $\rho(\mathbf{r})$ and $\rho_{c}(\mathbf{r})$ are very close to the values in bulk superfluid ${ }^{4} \mathrm{He}$, with $\rho_{c}(\mathbf{r}) \simeq$ $0.1 \rho(\mathbf{r})$. This indicates that even droplets of $N=70$ atoms are large enough to ensure that the surface region density profile will be very similar to that of a planar free surface of bulk superfluid ${ }^{4} \mathrm{He}$. This is confirmed by more recent calculations on larger droplets [9]. In the case of planar (vs spherical) symmetry, the atoms will Bose condense into states with zero momentum parallel to the surface [10].

Krotscheck 10 first found that the local condensate density $\rho_{c}(\mathbf{r})$ increased when the total density $\rho(\mathbf{r})$ decreased in liquid ${ }^{4} \mathrm{He}$ in contact with a wall. Campbell 11 has recently drawn attention to the results in Ref. [710] and discussed them in the context of the formal theory of off-diagonal long range order (ODLRO) in liquid ${ }^{4} \mathrm{He}$ droplets. Apart from these papers, Bosecondensation has been ignored in the literature on inhomogeneous Bose liquids at $T=0$ (for references, see [4] and [12]). Moreover, even in Refs. [7] 11], what is still missing is a physical interpretation of the numerical results for $\rho_{c}(\mathbf{r})$ at surfaces as evidence for (and relevance of) a low density Bose gas in which the condensate fraction can be $100 \%$, and the important implications of this new picture. It is this aspect which the present letter addresses.

The numerical results in Fig. 1 are consistent with the empirical formula given in Ref. [7],

$$
\rho_{c}(r)=\rho(r)\left[1-0.68 \frac{\rho(r)}{\rho_{B}}\right]^{2},
$$

where $\rho_{B}$ is the bulk or saturation density of superfluid 
${ }^{4} \mathrm{He}$ at $T=0$. It is easy to verify using (3) that the local condensate fraction $\rho_{c}(r) / \rho(r)$ smoothly increases from 0.1 to unity as $\rho(r)$ goes from $\rho_{B}$ to zero. One of the most interesting features shown by Fig. 1 is that $\rho_{c}(r)$ develops a maximum. Using (3), this occurs when $\rho(r) \simeq 0.5 \rho_{B}$ and corresponds to $\rho_{c}(r) \simeq 0.22 \rho_{B}$. Thus the results of Ref. [7] predict that in a certain region, the density of ${ }^{4} \mathrm{He}$ atoms which are Bose-condensed can be twice as large as the bulk value $\rho_{c} \simeq 0.1 \rho_{B}$ ! Of course, as $\rho(r)$ decreases to zero, so must $\rho_{c}(r)$. While the precise magnitude may depend on the approximate calculations used in [7], we believe that the increase in $\rho_{c}(r)$ as we go from the center of the ${ }^{4} \mathrm{He}$ drop is a real effect. As noted in Ref. [7], the initial increase in $\rho_{c}(r)$ shown in Fig. 1 for $\rho(r)<\rho_{B}$ is consistent with the calculated and observed decrease in $\rho_{c}$ in bulk liquid ${ }^{4} \mathrm{He}$ under pressure 13. Thus in superfluid ${ }^{4} \mathrm{He}, \partial \rho_{c}(\rho) / \partial \rho \simeq-0.3$ for $\rho$ close to $\rho_{B}$. In contrast, in a dilute hard sphere Bose gas, $\partial \rho_{c}(\rho) / \partial \rho \lesssim 1[5]$.

Independently of the numerical results in Refs. [7,9], it is easy to see that the general structure of the variational many-particle wavefunction which LPP and others [8,9,14 16] have used to treat the free surface of liquid ${ }^{4} \mathrm{He}$ already builds in the essential physics of a surface region which is completely Bose-condensed into one singleparticle state. In their simplest form, such wavefunctions are assumed to have the Feenberg form

$$
\Psi\left(\mathbf{r}_{1}, \ldots \mathbf{r}_{N}\right)=A e^{\left[-\frac{1}{2} \sum_{i<j} u\left(\mathbf{r}_{i}-\mathbf{r}_{j}\right)-\frac{1}{2} \sum_{i} t\left(\mathbf{r}_{i}\right)\right]}
$$

including one and two-particle correlations. The function $t(\mathbf{r})$ controls the shape of the density profile $\rho(\mathbf{r})$ associated with this wavefunction. It is easy to see that (4) can be rewritten in the form

$$
\Psi\left(\mathbf{r}_{1}, \ldots \mathbf{r}_{N}\right)=A \Psi_{\text {Jastrow }}\left(\mathbf{r}_{1}, \ldots \mathbf{r}_{N}\right) \Psi_{\text {gas }}\left(\mathbf{r}_{1}, \ldots r_{N}\right)
$$

where $\Psi_{\text {Jastrow }}$ alone describes a bulk liquid and

$$
\Psi_{\text {gas }}\left(\mathbf{r}_{1}, \ldots \mathbf{r}_{N}\right)=\prod_{i=1}^{N} \phi_{0}\left(\mathbf{r}_{i}\right)
$$

describes a Bose gas of $N$ atoms all occupying the same single-particle state given by $\phi_{0}(\mathbf{r})=e^{-\frac{1}{2} t(\mathbf{r})}$ (i.e., with $100 \%$ BEC). Choosing $u(\mathbf{r})$ and $t(\mathbf{r})$ appropriately, wavefunctions such as (4) allow one the variational freedom to describe atoms in the bulk region (where $\Psi_{\text {gas }} \simeq 1$ ) and in the low density surface region (note that when the atoms are far apart, one has $\Psi_{\text {Jastrow }} \simeq 1$ ). It is important to realize that $\phi_{0}(\mathbf{r})$ is not the single-particle "natural orbital" in (2), since the latter is defined relative to the complete wavefunction in (4). The interpretation of (4) as described above in (5) and (6) has not been emphasized in the previous literature on superfluid surfaces (see, however, Ref. [7]) but we think the present analysis clarifies the physics behind the complete BEC found in the surface region.
In the rest of this letter, we discuss how our new picture can be used to describe the surface region in a rigorous fashion using the field-theoretic analysis of an inhomogeneous Bose-condensed fluid [3,5]. In addition, we point out that this has important implications concerning density functional approaches used to calculate the surface properties of superfluid ${ }^{4} \mathrm{He}$ 《4, 17, 18.

The field-theoretical approach to Bose systems is based on the presence of the symmetry-breaking order parameter $\Phi(\mathbf{r}) \equiv\langle\hat{\psi}(\mathbf{r})\rangle$ as the anomalous average of the field operator $\hat{\psi}(\mathbf{r})$ (see Ch. 3 of Ref. 34). The local condensate density is given by $\rho_{c}(\mathbf{r})=|\Phi(\mathbf{r})|^{2}$. The equation for the order parameter can be obtained starting from the Heisenberg equation for the field operator

$$
-i \frac{\partial}{\partial t} \hat{\psi}(\mathbf{r})=\left[H^{\prime}, \hat{\psi}(\mathbf{r})\right]
$$

where $H^{\prime}=H-\mu N$ is the grand canonical Hamiltonian. By carrying out explicitly the commutator of (7) and taking the statistical average on the equilibrium state of the system one obtains the exact equation [6.19]

$$
\left[-\frac{\hbar^{2} \nabla^{2}}{2 m}-\mu\right] \Phi(\mathbf{r})+\int d \mathbf{r}^{\prime} v\left(\mathbf{r}-\mathbf{r}^{\prime}\right)\left\langle\hat{\psi}^{\dagger}\left(\mathbf{r}^{\prime}\right) \hat{\psi}\left(\mathbf{r}^{\prime}\right) \hat{\psi}(\mathbf{r})\right\rangle=0
$$

where $v\left(\mathbf{r}-\mathbf{r}^{\prime}\right)$ is the bare He-He interatomic potential. When applied to the homogeneous liquid, (8) provides an exact, non-trivial relationship between the chemical potential $\mu$ and the long range behaviour of the nondiagonal two-body density matrix of a Bose condensed system [20].

In the case of the free surface, the value of the chemical potential coincides with the bulk ${ }^{4} \mathrm{He}$ binding energy $(-7.15 \mathrm{~K}$ at $T=0)$. In this case, (8) can be used to investigate the behaviour of the order parameter $\Phi(\mathbf{r})$ when $\mathbf{r}$ is in the asymptotic, low density region far from the surface. Our reasoning is as follows. The dominant contribution to the integral in (8) comes from $\mathbf{r}^{\prime}$ in the bulk region, where $\rho\left(\mathbf{r}^{\prime}\right)$ is large. For this contribution, $\left|\mathbf{r}-\mathbf{r}^{\prime}\right|$ is large and the correlation function in $(8)$ is given by the asymptotically exact formula

$$
\left.\left\langle\hat{\psi}^{\dagger}\left(\mathbf{r}^{\prime}\right) \hat{\psi}\left(\mathbf{r}^{\prime}\right) \hat{\psi}(\mathbf{r})\right\rangle\right|_{\left|\mathbf{r}-\mathbf{r}^{\prime}\right| \rightarrow \infty}=\left\langle\hat{\psi}^{\dagger}\left(\mathbf{r}^{\prime}\right) \hat{\psi}\left(\mathbf{r}^{\prime}\right)\right\rangle\langle\hat{\psi}(\mathbf{r})\rangle=\rho\left(\mathbf{r}^{\prime}\right) \Phi(\mathbf{r}) .
$$

This may be viewed as a generalization of the PenroseOnsager result 21]

$$
\left.\left\langle\hat{\psi}^{\dagger}\left(\mathbf{r}^{\prime}\right) \hat{\psi}(\mathbf{r})\right\rangle\right|_{\left|\mathbf{r}-\mathbf{r}^{\prime}\right| \rightarrow \infty}=\left\langle\hat{\psi}^{\dagger}\left(\mathbf{r}^{\prime}\right)\right\rangle\langle\hat{\psi}(\mathbf{r})\rangle=\Phi^{*}\left(\mathbf{r}^{\prime}\right) \Phi(\mathbf{r}) .
$$

Using (9) in (8), we conclude that for $\mathbf{r}$ in the low density region, (8) reduces to

$$
\left\{-\frac{\hbar^{2} \nabla^{2}}{2 m}-\mu+\int d \mathbf{r}^{\prime} v\left(\mathbf{r}-\mathbf{r}^{\prime}\right) \rho\left(\mathbf{r}^{\prime}\right)\right\} \Phi(\mathbf{r})=0
$$


where the Hartree potential

$$
v_{H}(\mathbf{r})=\int d \mathbf{r}^{\prime} v\left(\mathbf{r}-\mathbf{r}^{\prime}\right) \rho\left(\mathbf{r}^{\prime}\right)
$$

describes the field at $\mathbf{r}$ due to the bulk region where the density is large. For such contributions, where $\left|\mathbf{r}-\mathbf{r}^{\prime}\right|$ is large, only the long-range attractive van der Waals' tail of $v\left(\mathbf{r}-\mathbf{r}^{\prime}\right)$ is important. For contributions to (8) when $\left|\mathbf{r}-\mathbf{r}^{\prime}\right|$ is small ( $i$. $e$. , when $\mathbf{r}^{\prime}$ is in the low surface region), one can use $\left\langle\hat{\psi}^{\dagger}\left(\mathbf{r}^{\prime}\right) \hat{\psi}\left(\mathbf{r}^{\prime}\right) \hat{\psi}(\mathbf{r})\right\rangle \simeq\left|\Phi\left(\mathbf{r}^{\prime}\right)\right|^{2} \Phi(\mathbf{r})$ since we are dealing with a region of complete BEC. Such contributions are precisely those kept in the standard GrossPitaevskii theory [22,5] and as usual one needs to include additional multiple scattering terms [5] which screen the hard core in $v\left(\mathbf{r}-\mathbf{r}^{\prime}\right)$ in $(8)$. However the details are not important here (see Ref. [23]) since the low density at $\mathbf{r}$ makes these contributions to (8) negligible compared to the ones discussed above. Similarly, while the manybody screening of the hard core is not explicitly included in (11) and (12), contributions to $v_{H}(\mathbf{r})$ when $\left|\mathbf{r}-\mathbf{r}^{\prime}\right|$ is small are negligible because of the low density at $\mathbf{r}$.

In summary, we conclude that (11) allows one to determine the exact asymptotic behavior of $\Phi(\mathbf{r})$ far from the surface of superfluid ${ }^{4} \mathrm{He}$ [23]. Interpreting (11) in physical terms, we see that the bulk of the liquid produces an effective field which acts to stabilize the dilute inhomogeneous gas in the surface region. The fact that the atoms in the low density region only feel this effective field but otherwise are not affected by their interactions explains why this region is fully Bose-condensed. Equations with the same Hartree-like structure as (11) arise in discussions of the binding energy of an impurity atom on the free surface of liquid ${ }^{4} \mathrm{He}$, based on variational wavefunctions 24.

For a planar surface centered at $z=0$, the asymptotic behavior of (12) reduces at large $z$ to $v_{H}(z)=-\rho_{B} \alpha / z^{3}$, where $\alpha$ only depends on the parameters of the He-He attractive potential [24]. Using this in (11), one finds that the order parameter has the form

$$
\Phi(z) \propto e^{-\left(A z+B / z^{2}\right)},
$$

where $A=\sqrt{\frac{2 m|\mu|}{\hbar^{2}}} \simeq 1 \AA^{-1}$ and $B=m \rho_{B} \alpha / 2 \hbar^{2} A \simeq$ $5 \AA^{2}$. The above discussion then suggests that the exponential decay [25] exhibited by the resulting density profile $\rho(z)=\rho_{c}(z)=|\Phi(z)|^{2}$ is a direct consequence of the complete BEC in the low density region, which occurs (see Fig. 1) for $\rho(z) \lesssim 0.1 \rho_{B}$. This behavior differs, for example, from the surface density profile of a Fermi liquid, where one finds a faster decay, of the form $\rho(z) \propto z^{-2} e^{-2 A z}[26$. Integrating (13) to find the total number of atoms in the region of essentially $100 \%$ BEC, it corresponds to an equivalent surface density of about $10^{14}$ atoms $/ \mathrm{cm}^{2}$.

Whatever the density is, the kinetic energy functional of a Bose-condensed system always has a contribution directly related to $\Phi(\mathbf{r})$ which is given by [5, 19]

$$
\begin{aligned}
\int d \mathbf{r} \Phi^{*}(\mathbf{r})\left[-\frac{\hbar^{2} \nabla^{2}}{2 m}\right] \Phi(\mathbf{r}) & =\int d \mathbf{r} \frac{\hbar^{2}}{2 m}\left|\nabla \sqrt{\rho_{c}(\mathbf{r})}\right|^{2} \\
& +\frac{1}{2} m \int d \mathbf{r} \rho_{c}(\mathbf{r}) \mathbf{v}_{s}^{2}(\mathbf{r})
\end{aligned}
$$

where $\Phi(\mathbf{r}) \equiv \sqrt{\rho_{c}(\mathbf{r})} e^{i S(\mathbf{r})}$ and $m \mathbf{v}_{s}(\mathbf{r}) \equiv \hbar \nabla S(\mathbf{r})$ is the local superfluid velocity. The expression in (14) is the origin of the kinetic energy term in (8) and (11).

The long-range coherence effects associated with superfluidity 19] are related to the local phase $S(\mathbf{r})$ of the complex (two-component) Bose order parameter $\Phi(\mathbf{r})$ defined above. In a situation like the one considered here, where the local condensate density $\rho_{c}(\mathbf{r})$ is rapidly decreasing normal to the surface, the superfluid flow properties may be quite different parallel and perpendicular to the surface. This clearly has experimental implications.

Phenomenological density functional theories used for inhomogeneous superfluid ${ }^{4} \mathrm{He}$ [17, 18, 4] always start from an energy functional of $\rho(\mathbf{r})$ alone, usually of the form

$$
H[\rho(\mathbf{r})]=\int d \mathbf{r} \frac{\hbar^{2}}{2 m}|\nabla \sqrt{\rho(\mathbf{r})}|^{2}+V_{\text {corr }}[\rho(\mathbf{r})] .
$$

The first term is the kinetic energy of an inhomogeneous ideal Bose gas with a given local density $\rho(\mathbf{r})$, while $V_{\text {corr }}[\rho(\mathbf{r})]$ is a phenomenological expression used to describe the effects of interactions. More precisely, the first term in (15) describes the energy of a fictitious liquid of $N$ atoms as if they all occupied the same single-particle state $\phi_{0}(\mathbf{r})=\sqrt{\rho(\mathbf{r}) / N}$, corresponding to $\Phi(\mathbf{r})=\sqrt{\rho(\mathbf{r})}$. A complete density functional theory of Bose-condensed liquids would involve functionals of both the local density $\rho(\mathbf{r})$ and the local order parameter $\Phi(\mathbf{r})$ 27]. In such a theory, the first term of (14) would naturally arise in place of the first term in (15), although we note that the two terms coincide in the important low density surface region since $\rho_{c}(\mathbf{r}) \simeq \rho(\mathbf{r})$.

It would be useful to have finite temperature path integral Monte Carlo calculations of $\rho_{c}(\mathbf{r})$ in the surface region of superfluid ${ }^{4} \mathrm{He}$ [28]. Physically, it seems clear that the surface region will Bose condense at the bulk superfluid transition temperature $T_{\lambda} \simeq 2.17 \mathrm{~K}$. The chemical potential is the same for the liquid and vapor in equilibrium and since it is large and negative $(\mu \simeq-7 \mathrm{~K}$ from 0 to $2 \mathrm{~K}$ ), the ${ }^{4} \mathrm{He}$ vapor can always be described as an ideal classical gas. Fig. 11 is only valid at low temperatures $(\lesssim 1 \mathrm{~K})$. As the temperature increases, the condensate density $\rho_{c}(\mathbf{r})$ will be increasingly depleted [3] and thus it will be a smaller component of the total local density $\rho(\mathbf{r})$, in both the bulk and surface regions. However we expect that $\rho_{c}(\mathbf{r})$ will still decrease exponentially in the surface region, while $\rho(\mathbf{r})$ will go over smoothly to the small but finite density of the ${ }^{4} \mathrm{He}$ vapor.

In conclusion, in this letter we have argued that the surface region of superfluid ${ }^{4} \mathrm{He}$ in self-bound phases can be described as an inhomogeneous dilute Bose gas which is completely Bose-condensed at $T=0$. Dramatic evidence for this "self-bound Bose gas" is given by the numerical calculations in Ref. [7]. We have noted that the 
asymptotic low density surface region can be described exactly by the order parameter $\Phi(\mathbf{r})$ given by the solution of a generalized Gross-Pitaevskii equation (11) taking full account of the long range van der Waals tail of the $\mathrm{He}-\mathrm{He}$ potential. Optical excitation of the surface atoms might be a way of detecting the completely Bose-condensed nature of the surface region of superfluid ${ }^{4} \mathrm{He}$. We have used our new physical ideas to suggest a more microscopic basis for the kinetic energy term used in density functional theories. This inhomogeneous Bose-condensed gas is readily available, and should complement the more exotic Bose gas systems on which much current research has concentrated [29].

We have had useful discussions concerning these results with F. Laloë and P. Nozières. A.G. would like to thank the Università di Trento for financial support and stimulating hospitality during a sabbatical leave. His research was also supported by NSERC of Canada.

* Permanent address : Department of Physics, University of Toronto, Toronto, Ontario, Canada, M5S 1A7.

[1] Bose-Einstein Condensation, ed. by A. Griffin, D. Snoke and S. Stringari (Cambridge, N.Y., 1995).

[2] For a review, see G. Taubes, Science 265, 184 (1994).

[3] A. Griffin, Excitations in a Bose-Condensed Liquid (Cambridge, N.Y., 1993).

[4] F. Dalfovo, A. Lastri, L. Pricaupenko, S. Stringari and J. Treiner, Phys. Rev. B52, 1193 (1995).

[5] A.L. Fetter and J.D. Walecka, Quantum Theory of Many-Particle Systems (McGraw-Hill, N.Y., 1971), p. $488 \mathrm{ff}$.

[6] P.C. Hohenberg and P.C. Martin, Annals of Physics 34, 291 (1965).

[7] D.S. Lewart, V.R. Pandharipande and S.C. Pieper, Phys. Rev. B37, 4950 (1988).

[8] The variational wavefunction $\Psi_{v}$ used in Ref. [7] is discussed in more detail in V.R. Pandharipande, S.C. Pieper and R.B. Wiringa, Phys. Rev. B34, 4571 (1986).

[9] S.A. Chin, J. Low Temp. Phys. 93, 921 (1993).

[10] E. Krotscheck, Phys. Rev. B32, 5713 (1985).

[11] C.E. Campbell, J. Low Temp. Phys. 93, 907 (1993). The "surface condensate" discussed here concerns a new kind of surface ODLRO which might arise in finite-sized droplets (an eigenvalue of $\mathrm{O}\left(N^{2 / 3}\right.$ ) instead of $\mathrm{O}(N)$ ). It is not related to the phenomenon discussed in the present letter.

[12] See, for example, E. Krotscheck, G.-X. Qian and W. Kohn, Phys. Rev. B31, 4245 (1985); S.A. Chin and E. Krotscheck, Phys. Rev. B45, 852 (1992); K.A. Gernoth, J.W. Clark, G. Senger and M.L. Ristig, Phys. Rev. B49, 15836 (1994).

[13] S. Stringari, J. Low Temp. Phys. 84, 279 (1991); Phys. Rev. B46, 2974 (1992).

[14] R.M. Bowley, J. Phys. C3, 2012 (1970).
[15] C.C. Chang and M. Cohen, Phys. Rev. A8, 3131 (1973).

[16] K.S. Liu, M.H. Kalos and G.V. Chester, Phys. Rev. B12, 1715 (1975).

[17] S. Stringari and J. Treiner, Phys. Rev. B36, 8369 (1987); J. Chem. Phys. 87, 5021 (1987).

[18] J. Dupont-Roc, M. Himbert, N. Pavloff and J. Treiner, J. Low Temp. Phys. 81, 31 (1990).

[19] P. Nozières and D. Pines, Theory of Quantum Liquids, Vol. II (Addison-Wesley, Redwood City, 1990), Ch. 10.

[20] See Eqs.(4) and (8) of first paper in Ref. [13].

[21] O. Penrose and L. Onsager, Phys. Rev. 104, 576 (1956).

[22] L.P. Pitaevskii, Sov. Phys.- JETP 13, 451 (1961); E.P. Gross, Nuovo Cimento 20, 454 (1961).

[23] One can prove that the correlation function in (8) is equal to $\rho\left(\mathbf{r}^{\prime}\right) \Phi(\mathbf{r})\left[1+F_{1}\left(\mathbf{r}, \mathbf{r}^{\prime}\right)\right]$, where the function $F_{1}$ takes into account all short-range correlations left out in (9). Using this result, one can easily see that (8) can be written quite generally in a form identical to (11) but with an inhomogeneous "screened" potential $v_{\text {eff }}\left(\mathbf{r}, \mathbf{r}^{\prime}\right) \equiv v\left(\mathbf{r}-\mathbf{r}^{\prime}\right)\left[1+F_{1}\left(\mathbf{r}, \mathbf{r}^{\prime}\right)\right]$. This formulation provides a rigorous basis for determining $\Phi(\mathbf{r})$ in regions where the density is non-negligible (see also Ref. [11]). In homogeneous fluids, the function $F_{1}$ has been extensively discussed by M.L. Ristig and J.W. Clark, Phys. Rev. B40, 4355 (1989) and Stringari [13].

[24] See, for example, I.B. Mantz and D.O. Edwards, Phys. Rev. B20, 4518 (1979).

[25] The leading order result $\rho(z) \sim e^{-2 A z}$ obtained here was first suggested by W.F. Saam, Phys. Rev. A4, 1278 (1971) and T. Regge, J. Low Temp. Phys. 9, 123 (1972).

[26] See Ref. 16 of E. Zaremba and W. Kohn, Phys. Rev. B15, 1769 (1977).

[27] A. Griffin, Can. Journ. Phys., in press.

[28] P. Sindzingre, M.L. Klein and D.M. Ceperley, Phys. Rev. Lett. 63, 160 (1989).

[29] Since this paper was submitted, essentially complete BEC has been achieved in a dilute gas of $\mathrm{Rb}$ atoms trapped in a harmonic potential well. See M.H. Anderson, J.R. Ensher, M.R. Matthews, C.E. Wieman and E.A. Cornell, Science 269, 198 (1995).

FIG. 1. The total density $\rho(r)$ and condensate density $\rho_{c}(r)$ as a function of the distance from the center of a ${ }^{4} \mathrm{He}$ droplet of 70 atoms, based on Fig. 5 of Ref. [7]. For simplicity, we have smoothed out the small oscillations in $\rho(r)$. 